\title{
Multiscale quantum/atomistic coupling using constrained density functional theory
}

\author{
Xu Zhang, ${ }^{1}$ Gang Lu, ${ }^{1, *}$ and W. A. Curtin ${ }^{2}$ \\ ${ }^{1}$ Department of Physics and Astronomy, California State University Northridge, Northridge, California 91330-8268, USA \\ ${ }^{2}$ Institute of Mechanical Engineering, Ecole Polytechnique Federale de Lausanne, Lausanne, Switzerland \\ (Received 20 November 2012; revised manuscript received 26 January 2013; published 28 February 2013)
}

\begin{abstract}
Multiscale coupling of a quantum mechanical $(\mathrm{QM})$ domain to a coarser-scale material description in a larger surrounding domain should yield forces and energies in the QM domain that are the same as would be achieved in a QM simulation of the entire system. Here, such a coupling is achieved by using constrained density functional theory (DFT) in which the quantum mechanical interaction between the domains is captured via a constraint potential arising from an imposed constraint on the charge density in a boundary region between the two domains. The implementation of the method, including the construction of the constraint charge density and the calculation of the constraint potential, is presented. The method is applied to problems in three different metals (Al, Fe, and Pd) and is validated against periodic DFT calculations. The method reproduces the QM charge density and magnetic moments of bulk materials, produces a reasonable edge dislocation core structure for $\mathrm{Fe}$, and also gives accurate vacancy formation energy for $\mathrm{Al}$ and chemisorption energy on a flat Pd surface. Finally, the method is used to study the chemisorption energy of CO on a stepped Pd surface. In general, the method can mitigate fictitious interactions between surface steps and other extended defects, and accommodate long-range deformation fields, and thus improves upon periodic DFT calculations.
\end{abstract}

DOI: 10.1103/PhysRevB.87.054113

PACS number(s): 71.15.Mb, 75.70.Rf, 68.43.Bc

\section{INTRODUCTION}

Despite ever increasing computational power, modeling and simulation of complex materials at the atomic level remains an enormous challenge. ${ }^{1}$ On one hand, although quantum mechanical $(\mathrm{QM})$ calculations are indispensable for treating chemical reactions, charge transfer, electron excitation, and magnetism in materials, they are often so expensive that no more than a few hundred atoms can be handled. On the other hand, atomistic simulations, molecular mechanics, or linear elasticity, generically all labeled as "MM" throughout this paper, based on empirical interatomic potentials, force fields, or elasticity, are usually capable of describing small deformations and electrostatic interactions at a much lower computational cost but are unable to deal with general chemical interactions. Therefore, the development of QM/MM multiscale computational methodologies has been pursued to achieve quantum mechanical level accuracy for problems that are cost prohibitive using conventional approaches. ${ }^{1-3}$ A large number of QM/MM applications exist in chemistry and biochemistry, ${ }^{3-5}$ where the system can be partitioned into $\mathrm{QM}$ and $\mathrm{MM}$ parts by cutting the chemical bonds linking the two domains and then saturating the dangling bonds at the boundary of the QM region by so-called link atoms. $^{3-5}$ This type of procedure is justifiable because of the localized nature of the chemical bonds in molecular systems. Far fewer QM/MM-like simulations have been attempted in metallic systems, ${ }^{6-10}$ where highly delocalized electrons and a long-ranged density matrix ${ }^{11}$ make capturing the $\mathrm{QM} / \mathrm{MM}$ interaction energy a challenge.

One emerging concept for accurate coupling in metals is self-consistent embedding theory. ${ }^{12-14}$ In this theory, the total charge density of the QM/MM system is decomposed into partial charge densities for the $\mathrm{QM}$ region and the MM region, and the $\mathrm{QM} / \mathrm{MM}$ interaction energy is formulated based on DFT. An embedding potential $v_{\text {emb }}$, defined as the functional derivative of the interaction energy with respect to the $\mathrm{QM}$ partial charge density, is then included in the self-consistent calculation of the QM region. $v_{\mathrm{emb}}$ is intended to capture the nonadditive kinetic energy ${ }^{15} T^{\text {nadd }}$ and the corresponding nonadditive kinetic potential $v_{\text {emb }}^{\text {nadd }}$ that have a fundamentally quantum mechanical origin, but require the approximation of orbital-free density functional theory (OFDFT). ${ }^{12-14,16-20}$ OFDFT is confined, to date, to the few materials, primarily main group elements, for which the approximate kinetic energy functionals and local pseudopotentials are sufficiently accurate. In this paper, we propose a quantum embedding theory based on constrained DFT that uses standard KohnSham (KS) DFT and can be applied to a much broader range of metallic materials.

In general, constrained DFT allows the ground-state energy to be determined self-consistently with an arbitrary density constraint by making an appropriate choice of the external potential. Using a Lagrange multiplier, a constraint potential is introduced to enforce the desired density constraint. ${ }^{21}$ Recent efforts strive to compute the exact $v_{\text {emb }}^{\text {nadd }}\left(\text { and/or } v_{\text {emb }}\right)^{22-25}$ by using the constraint concepts ${ }^{26-28}$ but all of these works require DFT calculations for the entire system, and so do not achieve the advantages of a multiscale $\mathrm{QM} / \mathrm{MM}$ simulation. The essence of the QM/MM method proposed here is to constrain the charge density in the QM/MM boundary region to an accurate target charge density that reflects the state of the material in the MM domain, and then determine the corresponding constraint potential to be applied to the QM region. This constraint method automatically includes the nonadditive kinetic potential while avoiding any artificial interface that leads an incorrect charge density and spurious ionic forces permeating into the QM region. The previous QM/MM method ${ }^{7,13}$ of two of the authors cannot include the nonadditive kinetic energy within a standard Kohn-Sham DFT method. The previous QM-CADD method $^{8}$ of another of the authors uses a relatively thick boundary region to prevent unwanted surface electronic relaxations from generating forces 
in the interior region, and is thus far more costly. The present QM/MM approach based on the constrained DFT is the first step toward eliminating the problems with these prior methods, leading to an efficient but robust multiscale method.

The remainder of this paper is organized as follows. In Sec. II, we introduce the general QM/MM method coupling formalism by defining the key concepts and physical quantities. In Sec. III, we discuss computational algorithms that allow the method to be implemented within standard Kohn-Sham DFT. In Sec. IV, we provide validations of the QM/MM method by applying it to three different types of metals including the main group ( $\mathrm{Al}$ ), nobel $(\mathrm{Pd})$, and magnetic transition $(\mathrm{Fe})$ metals. We examine the vacancy formation energy in $\mathrm{Al}$, the magnetic properties of bulk $\mathrm{Fe}$, and the dislocation structure of $\mathrm{Fe}$, in each case comparing to the results of standard periodic DFT calculations. We also calculate the chemisorption energy of a $\mathrm{CO}$ molecule on flat and stepped Pd surfaces as examples of potential applications of the method. Finally, we summarize our work in Sec. V.

\section{CONSTRAINED DFT QM/MM METHODOLOGY}

\section{A. Energies and forces}

The basic assumption of the QM/MM method is that the MM region is defect free and has small elastic deformations; strong lattice deformations and significant electron redistribution are contained only in the QM region. More specifically, the entire $\mathrm{QM} / \mathrm{MM}$ system is partitioned into three spatial domains labeled I, II, III, respectively, as shown schematically in Fig. 1. The inner QM Region I involves bond breaking/formation, chemical reaction, charge transfer phenomena, or other defects with topological changes in charge density, thus requiring quantum treatment. The outer MM Region III involves small deformations away from perfect crystallinity and so can be treated using atomistic interatomic potentials (or continuum mechanics via finite elements). QM Region II exists between the Regions I and III, and serves as a boundary region that couples Regions I and II; it must be similar to Region III with no defects and relatively small deformations. The selection of Region II must ensure that (i) there is no direct electronic bonding between Regions I and III, and (ii) the separation between Region I and Region III is greater than the interatomic potential cutoff distance. Hence, Regions I and III only interact through Region II. Typically, Region II includes two atomic layers of ions as shown in Fig. 1.

The total energy of the entire system can be formally written as

$$
\begin{aligned}
E_{\mathrm{tot}}[\mathrm{I}+\mathrm{II}+\mathrm{III}]= & E[\mathrm{I}]+E[\mathrm{II}]+E[\mathrm{III}] \\
& +E_{\text {int }}[\mathrm{I}, \mathrm{II}]+E_{\text {int }}[\mathrm{II}, \mathrm{III}],
\end{aligned}
$$

where the interaction energies are expressed as

$$
\begin{aligned}
E_{\mathrm{int}}[\mathrm{I}, \mathrm{II}] & =E[\mathrm{I}+\mathrm{II}]-E[\mathrm{I}]-E[\mathrm{II}] \\
E_{\mathrm{int}}[\mathrm{II}, \mathrm{III}] & =E[\mathrm{II}+\mathrm{III}]-E[\mathrm{II}]-E[\mathrm{III}],
\end{aligned}
$$

and we have expressly avoided denoting any specific methods used to compute all of these energies. Substituting the interaction energies into Eq. (1), we have

$$
E_{\mathrm{tot}}[\mathrm{I}+\mathrm{II}+\mathrm{III}]=E[\mathrm{I}+\mathrm{II}]+E[\mathrm{II}+\mathrm{III}]-E[\mathrm{II}] .
$$

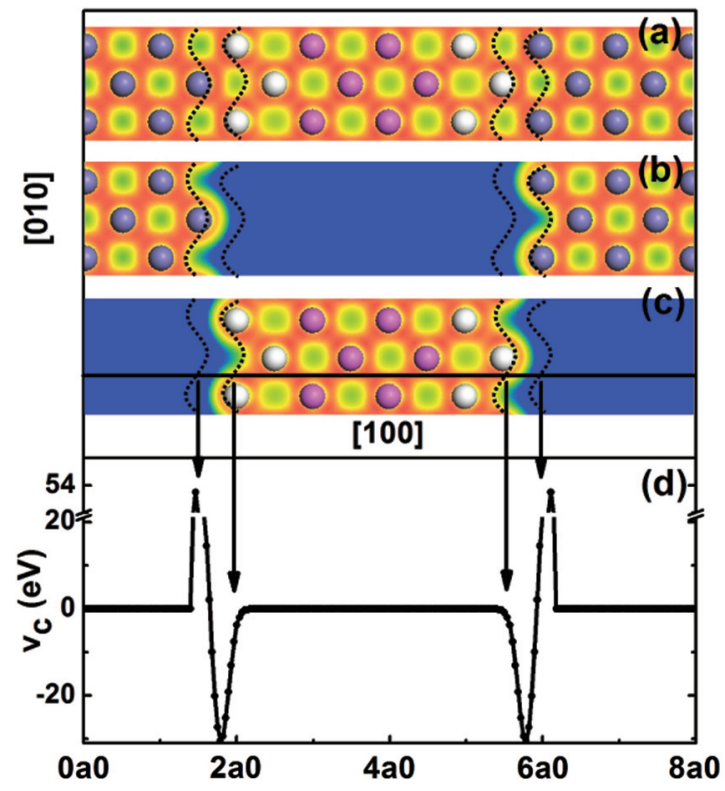

FIG. 1. (Color online) Partition of spatial domains and decomposition of charge densities in the QM/MM method by taking a perfect Al lattice as an example. The entire system (a) is partitioned into subsystems (b) and (c) in terms of both ions and electron densities. The magenta, white, and gray spheres represent the ions belonging to Regions I, II, and III, respectively. The dashed curves outline the boundaries of $\Omega_{\mathrm{c}}$ in (001) plane. The electron densities $\rho_{\mathrm{tot}}, \rho_{\mathrm{MM}}$, and $\rho_{\mathrm{QM}}$ in (001) plane are displayed in (a), (b), and (c), respectively, where the density contour scales (in $\AA^{-3}$ ) range from 0.0 (blue) to 0.24 (red). (d) The constraint potential is plotted along a straight line in [100] direction for a perfect Al lattice; the straight line is indicated in (c). All these results are obtained by $\lambda=20$ for Al.

Now we specify that the energy $E[\mathrm{I}+\mathrm{II}]$ will be computed using a QM DFT method, $E[\mathrm{I}+\mathrm{II}]=E^{\mathrm{DFT}}[\mathrm{I}+\mathrm{II}]$ and that the energy $E[\mathrm{II}+\mathrm{III}]$ will be computed using an MM method, $E[\mathrm{II}+\mathrm{III}]=E^{\mathrm{MM}}[\mathrm{II}+\mathrm{III}]$. The remaining energy $E[\mathrm{II}]$, subtracted out so as to avoid "double counting" of the energy of Region II, can be computed by either DFT or MM. Here, we compute $E[\mathrm{II}]$ using MM, $E[\mathrm{II}]=E^{\mathrm{MM}}[\mathrm{II}]$, so that the total energy retains QM energies from both Regions I and II. The $\mathrm{QM} / \mathrm{MM}$ method then yields an estimate for the total energy of the entire system as

$E_{\mathrm{tot}}[\mathrm{I}+\mathrm{II}+\mathrm{III}]=E^{\mathrm{DFT}}[\mathrm{I}+\mathrm{II}]+E^{\mathrm{MM}}[\mathrm{II}+\mathrm{III}]-E^{\mathrm{MM}}[\mathrm{II}]$.

The total energy thus requires one DFT computation over Regions I and II. Usually, a standard cluster computation of $E^{\mathrm{DFT}}[\mathrm{I}+\mathrm{II}]$ would lead to relaxation of the electronic degrees of freedom near the outer surface of the computational domain (i.e., in Region II), and propagation of density fluctuations (Friedel oscillations) deep into Region I. Such effects are partially mitigated in QM-CADD by using a wide Region II and a large "smearing parameter", ${ }^{8}$ both approximations that can be tuned and quantitatively evaluated but such an approach remains computationally intensive. Here, as described below, we use constrained DFT to compute $E^{\mathrm{DFT}}[\mathrm{I}+\mathrm{II}]$ and greatly suppress spurious surface effects in the solution for the electron density and energy in Region I. 
To obtain equilibrium structures requires computation of the ionic force $\mathbf{F}_{i}=-\partial E_{\text {tot }} / \partial \mathbf{R}_{i}$ on any ion $i$ that emerges from the above energy formulation. Forces on ions in Region I only arise from the first term, $E^{\mathrm{DFT}}[\mathrm{I}+\mathrm{II}]$, and thus are fully quantum mechanical in origin. Forces on ions in Region III arise only from the second term, $E^{\mathrm{MM}}[\mathrm{II}+\mathrm{III}]$, and thus are fully atomistic in origin. However, forces on ions in Region II arise from a combination of DFT and MM energies. Because the DFT and MM energy functionals have different nonlocal behaviors, an inconsistency arises that gives rise to so-called "ghost forces", ${ }^{29}$ a well-known problem in energy-based multiscale methods. Ghost forces are avoided by using a single method throughout the entire domain to compute the force in Region II. Here, MM is used to calculate the forces on Region II ions by $-\partial E^{\mathrm{MM}}[\mathrm{I}+\mathrm{II}+\mathrm{III}] / \partial \mathbf{R}_{\mathrm{II}}$. Given these forces, the system is driven to a stable equilibrium structure by minimizing the ionic forces on all ions in the system. Equation (4) then provides an estimate of the total system energy.

We reiterate that the $\mathrm{MM}$ domain could use a range of methods of varying accuracy; the main requirement of the MM method is that it provides accurate deformed ion positions in Regions II and III. For systems where suitable interatomic potentials are available, e.g., embedded atom method (EAM) ${ }^{30}$ potentials, the MM domain could use EAM. In general, the MM domain could use linear elasticity where the elastic constants and lattice constant correspond exactly to those computed by DFT on the material of interest. ${ }^{8}$ The MM domain could also use the Cauchy-Born rule applied to deformed DFT unit cell, as done in the quasicontinuum method and other QM/MM methods in the literature. ${ }^{29,31}$ The choice of an MM method is thus open and depends on the accuracy of available methods for the materials under study. In this paper, we will use the EAM method to perform calculations in the MM domain but this does not restrict the applicability of the general method.

The flowchart of the overall QM/MM method is shown in Fig. 2(a) illustrating the calculations of the total energy and forces. With the initial positions of all ions, the constrained DFT is performed for Region I and II to determine $E^{\mathrm{DFT}}[\mathrm{I}+\mathrm{II}]$
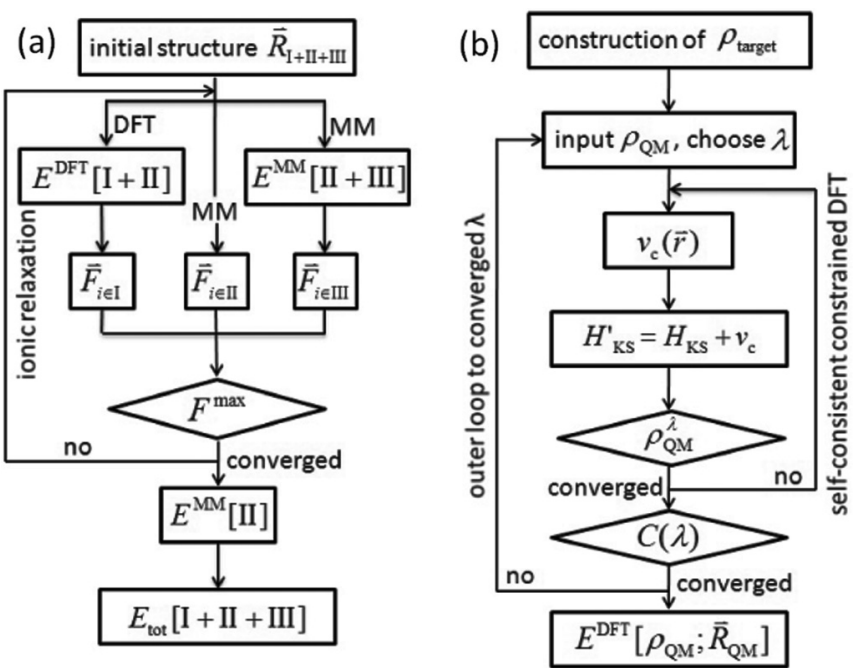

FIG. 2. (a) Flowchart of the QM/MM method in calculating the total energy and forces. (b) Flowchart of the constrained DFT calculation of Region I and II. and the forces on Region I ions. Two MM calculations (one for Region I + II + III and the other for Region II + III) are carried out to obtain the forces on Region II ions and $E[\mathrm{II}+\mathrm{III}]$. From $E[\mathrm{II}+\mathrm{III}]$, the forces on Region III ions are computed accordingly. The ionic relaxation is then performed until the forces are converged before the total energy $E_{\text {tot }}[\mathrm{I}+\mathrm{II}+\mathrm{III}]$ is calculated.

\section{B. Constrained DFT}

In the above, we have merely divided space and ions into domains in fairly standard manner. The computation of the DFT energies now needs to be specified, and requires a description of the electron density in the system. This is where we employ the constrained DFT. This QM/MM method will partition charge density as opposed to wave functions because "charge densities are nearsighted and wave functions are not." 32 The total electron density is decomposed into contributions associated with the QM ions in Regions I+ II and the MM ions in Region III, $\rho_{\text {tot }}=\rho_{\mathrm{QM}}+\rho_{\mathrm{MM}}$. The two electron densities $\rho_{\mathrm{QM}}$ and $\rho_{\mathrm{MM}}$ are not confined to their own spatial domains, i.e., $\rho_{\mathrm{QM}}$ extends into the $\mathrm{MM}$ region and vice versa. Thus, a domain $\Omega_{\mathrm{c}}$ is defined by the overlapping QM and MM charge densities, which straddles Regions II and III, as shown in Fig. 1. Because there is no direct electronic bonding between Regions I and III, the electron density from ions in Region $\mathrm{I}$ is required to be zero in $\Omega_{\mathrm{c}}$ to avoid overlap with $\rho_{\mathrm{MM}}$. Thus, $\rho_{\mathrm{QM}}\left(\mathbf{r} \in \Omega_{\mathrm{c}}\right)$ is due solely to electrons associated with the ions in Region II.

Since, relative to the perfect crystal, there are no drastic changes to the atomic structures in Regions II and III, their electron densities should be bulklike and can thus be well represented by a superposition of atomic charge densities, determined a priori for each ionic species. More specifically, the MM charge density is computed as the sum of atomic charge densities $\rho_{\mathrm{at}}\left(\mathbf{r}-\mathbf{R}_{i}\right)$ centered at each $\mathrm{MM}$ ion $\mathbf{R}_{i}$, $\rho_{\mathrm{MM}}(\mathbf{r})=\sum_{i \in \mathrm{III}} \rho_{\mathrm{at}}\left(\mathbf{r}-\mathbf{R}_{i}\right)$ where the index $i$ runs over all ions in the MM region. Similarly, we define a target charge density $\rho_{\text {target }}=\sum_{i \in \mathrm{II}} \rho_{\mathrm{at}}\left(\mathbf{r}-\mathbf{R}_{i}\right)$, where the index $i$ runs over all ions in Region II, which represents the bulklike electron density expected in Region II. The QM charge density $\rho_{\mathrm{QM}}$ is then obtained as the outcome of a constrained DFT calculation in which the charge density $\rho_{\mathrm{QM}}$ is constrained to match the predetermined target charge density $\rho_{\text {target }}$ within $\Omega_{\mathrm{c}}$, i.e., the density constraint is $\rho_{\mathrm{QM}}(\mathbf{r})=\rho_{\text {target }}(\mathbf{r})$ in $\Omega_{\mathrm{c}} \cdot \rho_{\text {target }}$ (and its corresponding constraint potential given below) thus provides a constraint for the determination of $\rho_{\mathrm{QM}}$ throughout the DFT domain of I + II $+\Omega_{c}$.

The constraint is not imposed on the entire Region II because the electron density of Region I extends into partial space of Region II and should not be constrained. The constraint is imposed only in $\Omega_{c}$, which is computed selfconsistently as the ionic positions are changed. Numerically, $\Omega_{\mathrm{c}}$ encompasses those points $\mathbf{r}$ satisfying

$$
\rho^{\prime}(\mathbf{r})>\rho_{\mathrm{cf}},
$$

where $\rho^{\prime}(\mathbf{r})=\min \left\{\rho_{\mathrm{QM}}(\mathbf{r}), \rho_{\mathrm{MM}}(\mathbf{r})\right\}$ and $\rho_{\mathrm{cf}}=10^{-4} \AA^{-3}$. In other words, $\Omega_{\mathrm{c}}$ is the region where the charge densities from both the $\rho_{\mathrm{QM}}$ and $\rho_{\mathrm{MM}}$ exceed the small value $\rho_{\mathrm{cf}}$, and is thus the region in which the two charge densities overlap. 
To determine $\Omega_{\mathrm{c}}, \rho_{\mathrm{QM}}$ is first constructed as a superposition of atomic charge densities centered at each QM ion; it also serves as the initial input charge density in the self-consistent DFT calculations.

Having specified the target charge density and the domain $\Omega_{\mathrm{c}}$ where the constraint is imposed, we now develop the associated constraint potential that is added as an "externally applied potential" to the KS equations to drive the groundstate charge density toward the target charge density in $\Omega_{\mathrm{c}}$. According to the Hohenberg and Kohn theorem, ${ }^{33}$ the constraint potential equals the embedding potential in the self-consistent embedding theory if the two external potentials yield the same ground state density $\rho_{\mathrm{QM}}$. We follow the method of Zhao et al. ${ }^{26}$ by defining the constraint potential as

$$
\tilde{v}_{\mathrm{c}}^{\lambda}(\mathbf{r})=\lambda \int_{\Omega_{\mathrm{c}}} \frac{\rho_{\mathrm{QM}}\left(\mathbf{r}^{\prime}\right)-\rho_{\text {target }}\left(\mathbf{r}^{\prime}\right)}{\left|\mathbf{r}-\mathbf{r}^{\prime}\right|} d \mathbf{r}^{\prime},
$$

where $\lambda$ is a penalty parameter. This constraint potential is essentially a penalty function associated with the total Coulomb potential generated by any differences between the target and actual electron densities. Since the constraint is only imposed in $\Omega_{\mathrm{c}}$, the constraint potential should be localized to $\Omega_{\mathrm{c}}$. Numerically, this is accomplished by multiplying $\tilde{v}_{\mathrm{c}}^{\lambda}$ by a cutoff function associated with the electron densities that define $\Omega_{\mathrm{c}}$ as

$$
w(\mathbf{r})= \begin{cases}1, & \text { if } \rho^{\prime}(\mathbf{r})>2 \rho_{\mathrm{cf}}, \\ \frac{\rho^{\prime}(\mathbf{r})}{\rho_{\mathrm{cf}}}-1, & \text { if } \rho_{\mathrm{cf}} \leqslant \rho^{\prime}(\mathbf{r}) \leqslant 2 \rho_{\mathrm{cf}}, \\ 0, & \text { if } \rho^{\prime}(\mathbf{r})<\rho_{\mathrm{cf}} .\end{cases}
$$

The constraint potential takes its full value if there is an overlap between the two densities and vanishes if either of the two densities is lower than $\rho_{\mathrm{cf}}$. The localized constraint potential is thus defined as

$$
v_{\mathrm{c}}^{\lambda}(\mathbf{r})=w(\mathbf{r}) \tilde{v}_{\mathrm{c}}^{\lambda}(\mathbf{r}) .
$$

For the collinear spin case, there are two constraint densities and two constraint potentials $v_{\mathrm{c}}^{\lambda \uparrow}$ and $v_{\mathrm{c}}^{\lambda \downarrow}$ acting on the spinup and spin-down electrons, respectively. $v_{\mathrm{c}}^{\lambda \uparrow}\left(v_{\mathrm{c}}^{\lambda \downarrow}\right)$ can be determined by Eq. (8) with the spin-up (spin-down) charge density $\rho_{\mathrm{QM}}^{\uparrow}\left(\rho_{\mathrm{QM}}^{\downarrow}\right)$ and $\rho_{\text {target }}^{\uparrow}\left(\rho_{\text {target }}^{\downarrow}\right)$.

The constraint potential $v_{\mathrm{c}}^{\lambda}$ parameterized by $\lambda$ is incorporated into the KS equation as an externally applied potential, so that

$$
\left[-\frac{1}{2} \nabla^{2}+v_{\mathrm{eff}}^{\mathrm{KS}}(\mathbf{r})+v_{\mathrm{c}}^{\lambda}(\mathbf{r})\right] \phi_{i}^{\lambda}(\mathbf{r})=\epsilon_{i}^{\lambda} \phi_{i}^{\lambda}(\mathbf{r}),
$$

where $v_{\text {eff }}^{\mathrm{KS}}(\mathbf{r})$ is the usual KS effective potential including electron-electron, electron-ion, and exchange-correlation potentials. For a given $\lambda, \rho_{\mathrm{QM}}^{\lambda}(\mathbf{r})$ is self-consistently determined by solving the KS equation. The resulting eigenvalues $\epsilon_{i}^{\lambda}$ and eigenfunctions $\phi_{i}^{\lambda}(\mathbf{r})$ with $\rho_{\mathrm{QM}}^{\lambda}(\mathbf{r})=\sum_{i} f_{i}\left|\phi_{i}^{\lambda}(\mathbf{r})\right|^{2}\left(f_{i}\right.$ is the occupation number) are then obtained. In the limit of $\lambda \rightarrow \infty, \rho_{\mathrm{QM}}^{\lambda}(\mathbf{r})$ would approach to $\rho_{\text {target }}(\mathbf{r})$ in $\Omega_{c}$. The energy of the $\mathrm{QM}$ region $E^{\mathrm{DFT}}[\mathrm{I}+\mathrm{II}]$ is thus calculated as

$$
\begin{aligned}
E^{\mathrm{DFT}}\left[\rho_{\mathrm{QM}}^{\lambda} ; \mathbf{R}_{\mathrm{QM}}\right]= & \sum_{i} f_{i} \epsilon_{i}^{\lambda}+E_{\mathrm{d} . c .}\left[\rho_{\mathrm{QM}}^{\lambda}\right]+\gamma_{\text {Ewald }}\left(\mathbf{R}_{\mathrm{QM}}\right) \\
& -\int_{\Omega_{\mathrm{c}}} \rho_{\mathrm{QM}}^{\lambda}(\mathbf{r}) v_{\mathrm{c}}^{\lambda}(\mathbf{r}) d \mathbf{r}
\end{aligned}
$$

where $E_{\text {d.c. }}$ is the usual double-counting energy term and $\gamma_{\text {Ewald }}$ is the Madelung energy. The energy contribution of the constraint potential (as an external potential) is subtracted from the constrained DFT energy because $E^{\mathrm{DFT}}\left[\rho_{\mathrm{QM}}^{\lambda} ; \mathbf{R}_{\mathrm{QM}}\right]$ represents the energy of the QM region by itself.

In this formulation, the constrained DFT forces the full QM electron density to match the target value in the overlap domain. The constrained DFT calculation includes the full nonadditive kinetic potential on the electrons associated with Regions I and II. If the target density were the exact density for the actual problem, the exact QM energy for Region I + II would then be obtained. The use of an approximate target density is thus the major approximation in the method. However, the use of any reasonable target density prevents the spurious relaxation of the electron density near the outer surfaces of the DFT domain, which is a major source of error in most other QM/MM methods.

\section{IMPLEMENTATION}

The constrained DFT calculations are performed using the Vienna $\mathrm{Ab}$ initio Simulation Package (VASP) ${ }^{34,35}$ with the projector augmented wave pseudopotentials. ${ }^{36}$ An energy cutoff of $300 \mathrm{eV}$ is used for the plane-wave basis set in all the three metals. The Perdew-Zunger local density approximation $(\mathrm{LDA})^{37}$ is used for the exchange-correlation (XC) functional of $\mathrm{Al}$ and the Perdew-Burke-Ernzerhof generalized gradient approximation (PBE-GGA) ${ }^{38}$ is employed for Fe and $\mathrm{Pd}$. The $k$-point sampling is based on the Monkhorst-Pack scheme, ${ }^{39}$ with details given in each case. The MM calculations employ the EAM potentials for $\mathrm{Al},{ }^{40} \mathrm{Fe},{ }^{41}$ and $\mathrm{Pd}^{42}$ but rescaled to yield the same lattice constant and bulk modulus as those of DFT for the given material. The DFT ionic relaxation is carried out with the conjugate-gradient algorithm and the force convergence criterion is $0.02 \mathrm{eV} / \AA$.

\section{A. Construction of $\rho_{\text {at }}$}

The target charge density is constructed as a sum of atomic-centered charge densities $\rho_{\mathrm{at}}\left(\mathbf{r}-\mathbf{R}_{i}\right)$ around each ion. As elaborated below, $\rho_{\mathrm{at}}$ is obtained for a perfect crystalline lattice of each element. The function $\rho_{\mathrm{at}}\left(\mathbf{r}-\mathbf{R}_{i}\right)$ must give an excellent representation of the true DFT charge density $\rho_{\text {bulk }}$ in the bulk perfect crystal. We thus determine $\rho_{\text {at }}$ by introducing a variational form and minimizing an objective function $I$, defined as the square of the difference between the target density and the true bulk density integrated over a unit cell of the perfect lattice, with respect to the variational parameters. The objective function $I$ is given by

$$
I=\int_{V}\left[\sum_{i} \rho_{\mathrm{at}}\left(\mathbf{r}-\mathbf{R}_{i}\right)-\rho_{\mathrm{bulk}}(\mathbf{r})\right]^{2} d \mathbf{r},
$$

where $V$ represents the volume of the unit cell and the sum includes all ions in a hypothetical large perfect crystal. The variational form for the atomiclike charge density is constructed from Gaussian-type orbitals as

$$
\rho_{\mathrm{at}}(\mathbf{r})=\sum_{l m} c_{l m}\left|R_{l}(r) Y_{l m}(\theta, \phi)\right|^{2},
$$


TABLE I. The parameters $c_{l m}$ and $\alpha_{l m}$ of $\rho_{\text {at }}(\mathbf{r})$ for Fe, Al, and Pd, respectively.

\begin{tabular}{|c|c|c|c|c|c|c|c|c|}
\hline \multirow[b]{2}{*}{$(l, m)$} & \multicolumn{4}{|c|}{$c_{l m}$} & \multicolumn{4}{|c|}{$\alpha_{l m}$} \\
\hline & $\mathrm{Fe}(\uparrow)$ & $\operatorname{Fe}(\downarrow)$ & $\mathrm{Al}$ & $\mathrm{Pd}$ & $\mathrm{Fe}(\uparrow)$ & $\mathrm{Fe}(\downarrow)$ & $\mathrm{Al}$ & $\mathrm{Pd}$ \\
\hline$(0,0)$ & 3.945 & 1.515 & 0.007 & 7.801 & 2.129 & 2.186 & 7.334 & 1.341 \\
\hline$(1,-1)$ & 0.0 & 0.0 & 0.998 & 0.0 & 0.0 & 0.0 & 0.620 & 0.0 \\
\hline$(1,0)$ & 0.0 & 0.0 & 0.998 & 0.0 & 0.0 & 0.0 & 0.620 & 0.0 \\
\hline$(1,1)$ & 0.0 & 0.0 & 0.998 & 0.0 & 0.0 & 0.0 & 0.620 & 0.0 \\
\hline$(2,-2)$ & 0.250 & 0.226 & - & 0.440 & 0.785 & 1.296 & - & 0.748 \\
\hline$(2,-1)$ & 0.250 & 0.226 & - & 0.440 & 0.785 & 1.296 & - & 0.748 \\
\hline$(2,0)$ & 0.201 & 0.355 & - & 0.440 & 0.924 & 0.582 & - & 0.748 \\
\hline$(2,1)$ & 0.250 & 0.226 & - & 0.440 & 0.785 & 1.296 & - & 0.748 \\
\hline$(2,2)$ & 0.201 & 0.355 & - & 0.440 & 0.924 & 0.582 & - & 0.748 \\
\hline
\end{tabular}

where $\{r, \theta, \phi\}$ are spherical coordinates, and $l$ and $m$ are angular and magnetic quantum numbers. $c_{l m}$ are coefficients ensuring that $\sum_{l m} c_{l m}$ equals the number of valence electrons of the atom. The $Y_{l m}$ are the spherical harmonic functions, and the radial functions $R_{l}$ are Gaussian functions given by

$$
R_{l}(r)=r^{l} A\left(l, \alpha_{l m}\right) \exp \left(-\alpha_{l m} r^{2}\right),
$$

where the $A(l, \alpha)$ are normalization factors given by

$$
A(l, \alpha)=\frac{\alpha^{(2 l+3) / 4}}{\sqrt{\pi} 2^{(1-(2 l+3) / 2) / 2} \sqrt{\Gamma((2 l+3) / 2)}} .
$$

The parameters $c_{l m}$ and $\alpha_{l m}$ are determined by minimizing $I$. In a collinear spin-polarized system such as Fe, there are two sets of atomiclike charge density $\rho_{\text {at }}^{\uparrow}(\mathbf{r})$ and $\rho_{\text {at }}^{\downarrow}(\mathbf{r})$ for spin-up and spin-down, respectively. The $\rho_{\text {at }}^{\uparrow}$ and $\rho_{\text {at }}^{\downarrow}$ are obtained by the minimization of the objective function using the bulk charge densities $\rho_{\text {bulk }}^{\uparrow}$ and $\rho_{\text {bulk }}^{\downarrow}$, respectively.

We have constructed $\rho_{\text {at }}$ for three metals, body-centered cubic (bcc) Fe, face-centered cubic (fcc) Al and fcc Pd, and their parameters are listed in Table I. Although the target charge density matches the bulk charge density very well overall, as
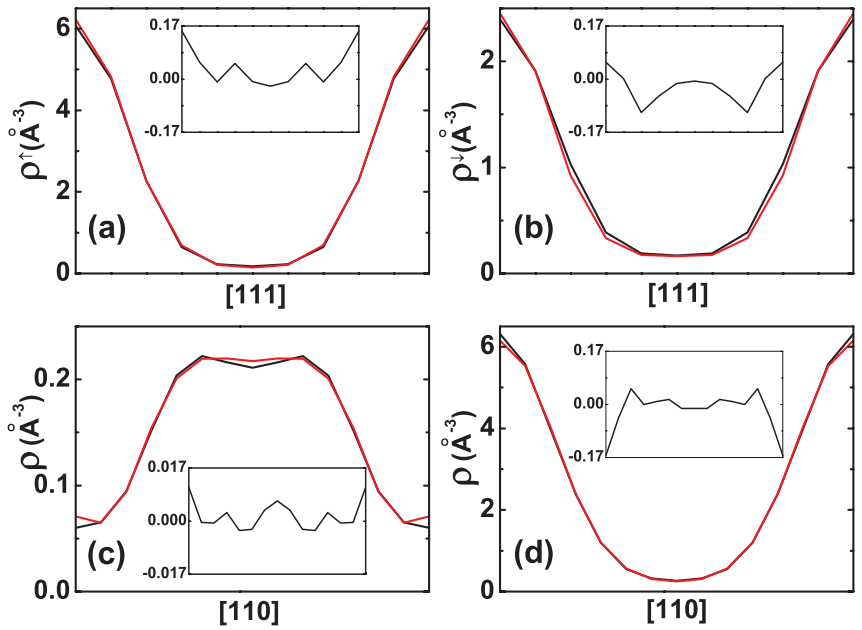

FIG. 3. (Color online) Comparisons between $\rho_{\text {bulk }}(\mathbf{r})$ obtained by the periodic DFT calculation (black curve) and the superposition of the atomic densities $\sum_{i} \rho_{\mathrm{at}}\left(\mathbf{r}-\mathbf{R}_{i}\right)$ (red curve) for (a) $\mathrm{Fe}(\uparrow)$, (b) $\mathrm{Fe}(\downarrow)$, (c) $\mathrm{Al}$, and (d) $\mathrm{Pd}$, respectively. Insets: the difference between $\rho_{\text {bulk }}(\mathbf{r})$ and $\sum_{i} \rho_{\text {at }}\left(\mathbf{r}-\mathbf{R}_{i}\right)$. shown in the Fig. 3, errors (displayed in the insets) do exist and they are the main source of error in the present QM/MM method. One measure of the quality of these charge densities is their predictions of the material properties in the perfect bulk lattice. By applying the constrained DFT formalism, we have calculated the lattice constant, bulk modulus, and cohesive energy of a cubic unit cell using the atomic densities thus generated. Table II shows the results for each of the three metals $\mathrm{Al}, \mathrm{Fe}$, and $\mathrm{Pd}$, as compared to the values obtained from a standard periodic DFT calculation. Overall, the differences in these properties between the bulk charge density and superposition of atomic charge densities are small. These differences are not a fundamental problem with the method, and can be mitigated by more sophisticated fitting procedures for the atomic charge density.

We note that by using a fixed $\rho_{\text {at }}(\mathbf{r})$ attached to each ion site, the present implementation does not permit the atomic charge density to relax as a function of lattice deformation. More specifically, although the first-order change in the solid charge density associated with the changing ion positions is captured, higher-order changes in the density are not captured. This effect is partially reflected in the computed bulk modulus of the materials, as shown in Table II, which are larger than the true QM values due to the lack of electronic relaxation. To remedy errors associated with this lack of electronic relaxation, we can envision generating a target charge density that depends on the deformation gradient $\mathbf{F}$ but we have not yet explored this avenue.

\section{B. Periodic DFT cell}

Computation of any DFT problems are facilitated by the use of a plane-wave basis. In the KS-DFT plane-wave calculation of the QM region, a periodic DFT cell is introduced over which the periodic boundary conditions (PBCs) are imposed. The PBCs are necessary for various fast Fourier transforms (FFTs), which are crucial for efficient numerical calculations. In our constrained DFT method, the constraint potential acts as an energetic barrier that prevents electrons in the DFT computation from moving outside of the domain of Regions $\mathrm{I}+\mathrm{II}+\Omega_{\mathrm{c}}$. Beyond $\Omega_{\mathrm{c}}$, the QM charge density as well as the wave functions are required to be zero. Thus, we can use any convenient periodic cell that encompasses the domain of Regions $\mathrm{I}+\mathrm{II}+\Omega_{\mathrm{c}}$. As long as the vacuum introduced 
TABLE II. Lattice constant, bulk modulus, and cohesive energy calculated based on the bulk charge density $\rho_{\text {bulk }}$ (labeled by "bulk") and the superposition of the fitted atomic densities $\sum_{i} \rho_{\mathrm{at}}\left(\mathbf{r}-\mathbf{R}_{i}\right)$ (labeled by "superposition").

\begin{tabular}{lccccccc}
\hline \hline & \multicolumn{3}{c}{ bulk } & & \multicolumn{3}{c}{ superposition } \\
\cline { 2 - 4 } \cline { 6 - 8 } & $\mathrm{Fe}$ & $\mathrm{Al}$ & $\mathrm{Pd}$ & & $\mathrm{Fe}$ & $\mathrm{Al}$ & $\mathrm{Pd}$ \\
\hline$a_{0}(\AA)$ & 2.833 & 3.982 & 3.955 & & 2.826 & 3.981 & 3.956 \\
$B(\mathrm{GPa})$ & 197 & 84 & 171 & & 207 & 89 & 171 \\
$E(\mathrm{eV})$ & -16.63 & -16.79 & -20.72 & & -16.68 & -16.77 & -20.70 \\
\hline \hline
\end{tabular}

between $\Omega_{\mathrm{c}}$ and the periodic cell is large enough, it can effectively eliminate the fictitious interaction between the periodic images. The use of PBCs with the realization that the real QM/MM system is nonperiodic is justified. Smaller cells are computationally more efficient, and so we use a cell that extends out about $5 \AA$ beyond the boundary of $\Omega_{\mathrm{c}}$. As a reminder, the DFT cell remains coupled to a surrounding MM region, so that long-range deformations are captured accurately in any problem.

\section{Evaluation of $\tilde{v}_{\mathrm{c}}^{\lambda}$}

In the DFT simulations, FFTs are employed in the calculation of the Coulomb integrals. However, the evaluation of $\tilde{v}_{\mathrm{c}}^{\lambda}$ in Eq. (6) presents a difficulty associated with the charge neutrality requirement of FFTs. This is because the integration $\int_{\Omega_{\mathrm{c}}}\left[\rho_{\mathrm{QM}}(\mathbf{r})-\rho_{\text {target }}(\mathbf{r})\right] d \mathbf{r}$ is not guaranteed to be zero, leading to potential numerical problems. To circumvent this problem, we define a surrogate target charge density $\rho_{\text {target }}^{*}(\mathbf{r})=$ $\sum_{i} \rho_{\mathrm{at}}\left(\mathbf{r}-\mathbf{R}_{i}\right)$, where the index $i$ runs over the entire QM region (Regions I and II). This modification ensures that $\rho_{\text {target }}^{*}(\mathbf{r})=\rho_{\text {target }}(\mathbf{r})$ in $\Omega_{\mathrm{c}}$ and $\int_{\Omega}\left[\rho_{\mathrm{QM}}(\mathbf{r})-\rho_{\text {target }}^{*}(\mathbf{r})\right] d \mathbf{r}=0$ where integration is over the entire space $\Omega$. We can then calculate $\tilde{v}_{\mathrm{c}}^{\lambda}$ as

$$
\tilde{v}_{\mathrm{c}}^{\lambda}(\mathbf{r})=\lambda \int_{\Omega} \frac{\rho_{\mathrm{QM}}\left(\mathbf{r}^{\prime}\right)-\rho_{\text {target }}^{*}\left(\mathbf{r}^{\prime}\right)}{\left|\mathbf{r}-\mathbf{r}^{\prime}\right|} d \mathbf{r}^{\prime},
$$

and the FFTs can be used without problems. The constraint potential is then localized to the domain of $\Omega_{\mathrm{c}}$ using Eq. (8). Comparing Eq. (15) to Eq. (6), Eq. (15) has additional contributions from space $\Omega-\Omega_{\mathrm{c}}$ that could induce errors in $\tilde{v}_{\mathrm{c}}^{\lambda}$. Since $\int_{\Omega-\Omega_{\mathrm{c}}}\left[\rho_{\mathrm{QM}}(\mathbf{r})-\rho_{\text {target }}^{*}(\mathbf{r})\right] d \mathbf{r}$ vanishes when the constrained DFT calculation is converged, this additional term also vanishes to the first order because the long-range Coulomb potential is zero in a charge-neutral system. Furthermore, as shown below, the numerical errors in using Eq. (15) turn out to be rather small, and the constraint potential itself does not contribute to the total energy, which is subtracted from the DFT computed energy as in Eq. (10).

\section{Choice of $\lambda$}

A constrained DFT calculation is converged if the resultant charge density is identical to the target charge density. In principle, Eq. (9) should be solved in the limit $\lambda=\infty$, but in practice, as $\lambda$ increases, the ability to enforce the constraint is swamped by other numerical factors so that convergence cannot be obtained. ${ }^{27}$ To circumvent this problem, one could solve Eq. (9) self-consistently with a series of larger and larger $\lambda$ values and then extrapolate the results to $\lambda=\infty .^{26}$
However, when a QM region contains hundreds of atoms this method becomes impractical. In addition, we find that the selfconsistent loops become increasingly difficult to converge with larger $\lambda$ values. Our aim is to perform single self-consistent KS calculations with a sufficiently large value of $\lambda$ that balances computational accuracy and efficiency.

To identify a suitable value for $\lambda$, we introduce the $\lambda$ dependent quantity

$$
C(\lambda)=\frac{1}{2 \lambda} \int_{\Omega_{\mathrm{c}}}\left[\rho_{\mathrm{QM}}^{\lambda}(\mathbf{r})-\rho_{\text {target }}(\mathbf{r})\right] v_{\mathrm{c}}^{\lambda}(\mathbf{r}) d \mathbf{r}
$$

to quantify the difference between the resultant and target charge densities, weighted by the constraint potential over the constrained domain. $C(\lambda)$ decreases with increasing $\lambda$ and vanishes when $\lambda \rightarrow \infty$. To determine optimal $\lambda$, we have carried out QM/MM calculations for a perfect bulk cell of each metal of dimensions $14 a_{0} \times 14 a_{0} \times a_{0}$ in $x, y$, and $z$ directions, respectively, where $a_{0}$ is the equilibrium lattice constant. The innermost $4 a_{0} \times 4 a_{0} \times a_{0}$ is the QM Region I+ II, containing 64 atoms for $\mathrm{Al}$ and $\mathrm{Pd}$ and 32 atoms for $\mathrm{Fe}$, and the remainder is the MM Region III. The constrained DFT calculations for Region $\mathrm{I}+\mathrm{II}+\Omega_{c}$ are performed in a rectangular box with dimensions $6 a_{0} \times 6 a_{0} \times$ $a_{0}$, with a vacuum (size of $2.5 a_{0}$ ) in the $x$ and $y$ directions and periodic boundaries in the $z$ direction, and using a $1 \times 1 \times 9$ $k$-point mesh.

The evaluated $C(\lambda)$ as a function of $\lambda$ are shown in Fig. 4. In addition to $C(\lambda)$, we also present the variation of the magnetic moments in Fe versus $\lambda$, which is another indication for accuracy of the charge density. For Al, higher accuracy $[\sim 0.05 \mathrm{eV}$ in $C(\lambda)]$ can be achieved by choosing a large $\lambda$ $(\sim 20)$, and no difficulties with achieving self-consistency are encountered. On the other hand, the difficulty in obtaining self-consistency for $\mathrm{Fe}$ and Pd prevents the use of such large values for $\lambda$. However, we find good convergence for $\lambda=2$ in $\mathrm{Fe}$, and we choose the largest practical value of $\lambda=5$ for Pd; these values balance accuracy and efficiency. The chosen $\lambda$ value for Fe also yields sufficiently accurate magnetic moments for Fe, as shown in Fig. 4(d).

The value of $\lambda$ constitutes the remaining source of error in the QM/MM method. Note that the value of $C(\lambda)$ does not represent any specific energy error in our implementation; it is only a measure of the penalty imposed for not attaining the target charge density. The energy contribution of the constraint potential is subtracted from the total energy, so that the error in the DFT calculation is associated with the small differences in charge density between the resultant and target values. The flowchart of the constrained DFT calculation involving the selection of $\lambda$ is displayed in Fig. 2(b). 

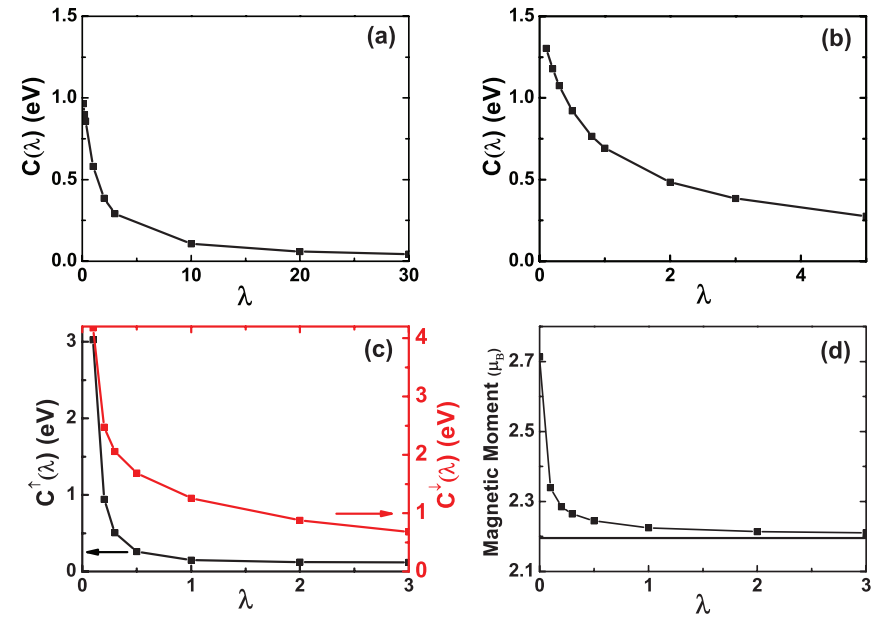

FIG. 4. (Color online) $C(\lambda)$ versus $\lambda$ for three ideal systems (a) Al, (b) Pd, (c) Fe. In (c), the black (red) curve represents the spin-up (spin-down) electrons. (d) The variation of the magnetic moment per atom as a function of $\lambda$ for Fe. The horizontal line indicates the magnetic moment of a perfect lattice determined from the periodic DFT calculation.

\section{VALIDATIONS AND APPLICATIONS}

In the following, we validate the QM/MM method against stand-alone periodic DFT calculations in the three metals of $\mathrm{Al}, \mathrm{Fe}$, and $\mathrm{Pd}$. In the validations, the periodic DFT results are taken as the reference against which we compare the QM/MM results whenever appropriate.

\section{A. Constraint potential and vacancy formation energy in Al}

$\rho_{\mathrm{QM}}, \rho_{\mathrm{MM}}$, and $\rho_{\text {tot }}$ are displayed in Fig. 1 for the perfect Al lattice. Although there is no visible discontinuity of charge density at the QM/MM boundary, the charge density errors across the boundary nonetheless exist, typically by a few percent. The charge density errors induce force errors on the interior QM ions. For the perfect Al lattice, we find a maximum force error of $0.025 \mathrm{eV} / \AA$. One can increase $\lambda$ and/or construct a better $\rho_{\text {target }}$ to systematically reduce the force errors.

We also plot the constraint potential $v_{\mathrm{c}}^{\lambda}(\mathbf{r})$ with $\lambda=20$ along a straight line in Fig. 1(d). The arrows indicate the overlap between $\rho_{\mathrm{QM}}$ and $\rho_{\mathrm{MM}}$ in $\Omega_{\mathrm{c}}$. The constraint potential consists of three parts: (i) the repulsive (positive) potential constraining $\rho_{\mathrm{QM}}$ and the wave functions in the QM region; it plays the similar role as the kinetic energy contribution in the embedding potential. $^{22}$ (ii) The attractive (negative) potential at the QM/MM boundary renders appropriate bonding between the QM and MM atoms. (iii) the electrons in Region I and the inner part of Region II are not constrained and thus experience zero constraint potential.

For the simplest validation of the QM/MM energetics, we calculate the vacancy formation energy in $\mathrm{Al}$ using both the QM/MM method and a stand-alone DFT. In the stand-alone DFT calculation, we use a periodic supercell consisting of 256 atoms $\left(4 a_{0} \times 4 a_{0} \times 4 a_{0}\right)$ with a $k$-point mesh of $3 \times 3 \times$ 3 . The DFT supercell is large enough to obtain the accurate single-vacancy formation energy. The vacancy formation energy is defined as

$$
E_{f v}^{(a)}=E^{(a)}(N-1)+E^{(a)}(1)-E^{(a)}(N) .
$$

The subscripts $(a)$ and $(b)$ denotes the stand-alone DFT and the QM/MM calculations, respectively. $E^{(a)}(N-1)$ and $E^{(a)}(N)$ are the total energy for the periodic lattice with and without a vacancy, respectively, and $E^{(a)}(1)=E^{(a)}(N) / N$ is the energy of single $\mathrm{Al}$ atom in the bulk. The calculated vacancy formation energy $E_{f v}^{(a)}$ is $0.75 \mathrm{eV}$. Using the same exchange-correlation functional, others have reported a value of $0.78 \mathrm{eV}{ }^{43}$ In the QM/MM calculations, the entire system consists of $14 a_{0} \times$ $14 a_{0} \times 14 a_{0}$ (10976 atoms) with the innermost $4 a_{0} \times 4 a_{0} \times$ $4 a_{0}$ (256 atoms) as the QM region. Only the $\Gamma$ point is used in the constrained DFT calculation with a vacuum placed in all three directions. The QM/MM vacancy formation energy is defined as

$$
E_{f v}^{(b)}=E^{(b)}(N-1)+E^{(a)}(1)-E^{(b)}(N),
$$

where $E^{(b)}(N-1)$ and $E^{(b)}(N)$ are the total energy for the entire system with and without a vacancy. The calculated vacancy formation energy $E_{f v}^{(b)}$ is $0.79 \mathrm{eV}$, which is in a good agreement with the stand-alone DFT values $(0.75 \sim 0.78 \mathrm{eV})$. We have performed an additional QM/MM calculation with a larger QM region, $4.5 a_{0} \times 4.5 a_{0} \times 4.5 a_{0}$ of 365 atoms, but obtained the same value $(0.79 \mathrm{eV})$ of $E_{f v}^{(b)}$. We have also calculated $E_{f v}^{(b)}$ for $\lambda=10$ and 30 , and determined the vacancy formation energy to be $0.81 \mathrm{eV}$ and $0.79 \mathrm{eV}$, respectively. Thus, $\lambda=20$ is sufficient to obtain the converged vacancy formation energy of Al. The discrepancy to the stand-alone results is probably due to the error in constructing the target charge density. Note that this single-vacancy example only serves the purpose of energetic validation of the QM/MM method; the QM/MM method offers no advantage over the periodic DFT in this case owing to the short-ranged strain field of a single vacancy.

\section{B. Magnetic moment in $\mathrm{Fe}$}

For transition metal Fe, we focus on the validation of the magnetic moments by considering the following systems: a perfect bulk lattice and a bulk with a self-interstitial atom (SIA). In the stand-alone DFT calculation of a SIA, a periodic supercell of $4 a_{0} \times 4 a_{0} \times 4 a_{0}$ consisting of 129 atoms is used with a $k$-point mesh of $3 \times 3 \times 3$. In the $\mathrm{QM} / \mathrm{MM}$ calculation of a SIA, an entire system of $14 a_{0} \times 14 a_{0} \times 14 a_{0}$ with an innermost QM region of $4 a_{0} \times 4 a_{0} \times 4 a_{0}$ is modeled. The calculation is performed at the $\Gamma$ point only. For both systems, the QM/MM results are compared to those of the stand-alone periodic DFT calculation in Figs. 5(a) and 5(c), respectively. Overall, there is an excellent agreement between the QM/MM and the periodic DFT calculations for the magnetic moments. To provide a reference point for comparison, we also display the difference in the magnetic moments between a DFT cluster calculation and the same stand-alone periodic DFT calculation in Figs. 5(b) and 5(d), respectively. The cluster refers to an isolated QM region without the surrounding MM atoms. It is striking how effectively the QM/MM method can cut down the magnetic moment errors at the cluster surfaces. Since the majority of QM/MM methods involve cluster calculations, 


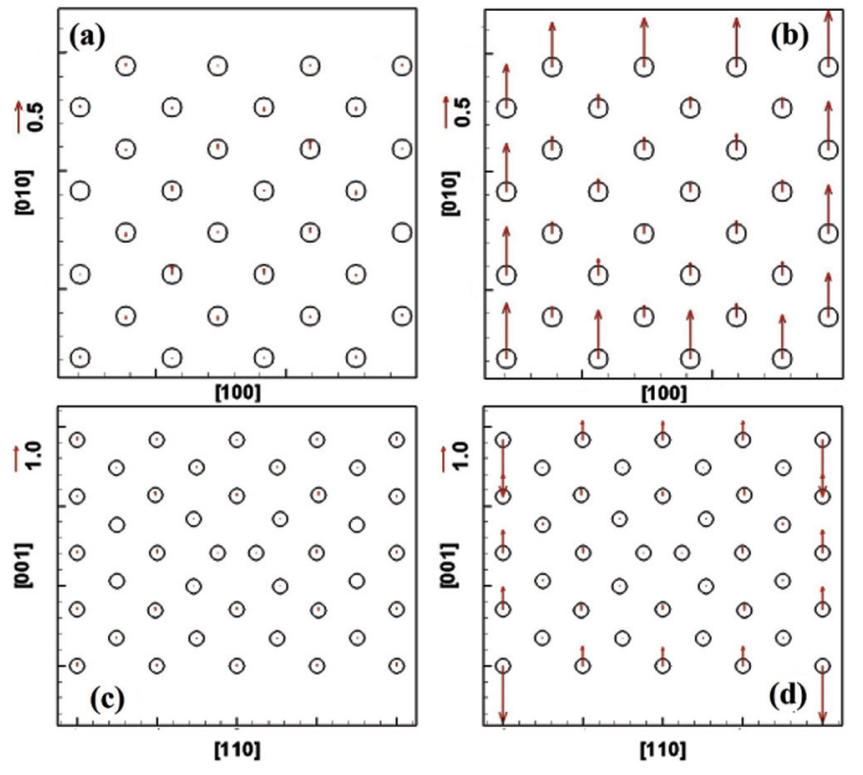

FIG. 5. (Color online) (a), (b) Difference in magnetic moments (in units of $\mu_{\mathrm{B}}$ and shown as arrows on each ion) between a periodic DFT calculation and (a) the QM/MM calculation, $\mu_{\mathrm{QM} / \mathrm{MM}}-\mu_{\text {period }}$, and (b) a cluster calculation, $\mu_{\text {cluster }}-\mu_{\text {period }}$ for the perfect Fe lattice; (c), (d) Difference in magnetic moments between a periodic DFT calculation and (c) the QM/MM calculation and (d) a cluster calculation for a self-interstitial in Fe.

the results shown here clearly demonstrate the superiority of the constrained QM/MM method relative to the other $\mathrm{QM} / \mathrm{MM}$ methods for magnetic materials.

\section{Edge dislocation in $\mathrm{Fe}$}

Here, we demonstrate the advantage of the QM/MM method over the periodic DFT in modeling an edge dislocation in Fe. The dislocation has a Burgers vector $\mathbf{b}=\frac{1}{2}\langle 111\rangle$ on $\{110\}$ slip plane. In the QM/MM simulation, the entire system consists of $202 \AA \times 202 \AA \times 6.93 \AA$ in [111], [1110], and [ $\overline{1} \overline{1} 2]$ directions, respectively, with 25398 atoms in total. Fixed boundary conditions are applied along [111] and [110] directions with the boundary displacement determined by the isotropic elastic solution of the edge dislocation. The dislocation line is along [ $1 \overline{1} 12]$ direction in which the periodical boundary condition is applied. The dimensions of the QM region are $21 \AA \times 10 \AA \times 6.93 \AA$ with 153 atoms, and the rest of the system belongs to the MM region. In the QM/MM calculation, the boundary QM region includes three atomic layers of QM ions in [111] direction and one atomic layer in [110] direction. Three [111] planes were included in the DFT calculation to ensure that there is no direct interaction between the interior QM atoms and the EAM atoms. The core structure of the edge dislocation is depicted in Fig. 6(a) . An rough average strain measure is computed for each atomic position $i$ as $\epsilon_{i}=\frac{1}{N_{\mathrm{nn}}} \sum_{j} \frac{r_{i j}-r_{i j}^{0}}{r_{i j}^{0}}$ and is shown in the contour plot of Fig. 6(a), where $i, j$ is the atom index and $j$ sums over the nearest neighbors of the atom $i ; N_{\mathrm{nn}}$ is the number of the nearest neighbors. $r_{i j}$ and $r_{i j}^{0}$ are the interatomic distances in the dislocation and the perfect lattice, respectively. In Fig. 6(b), we display the constraint potential

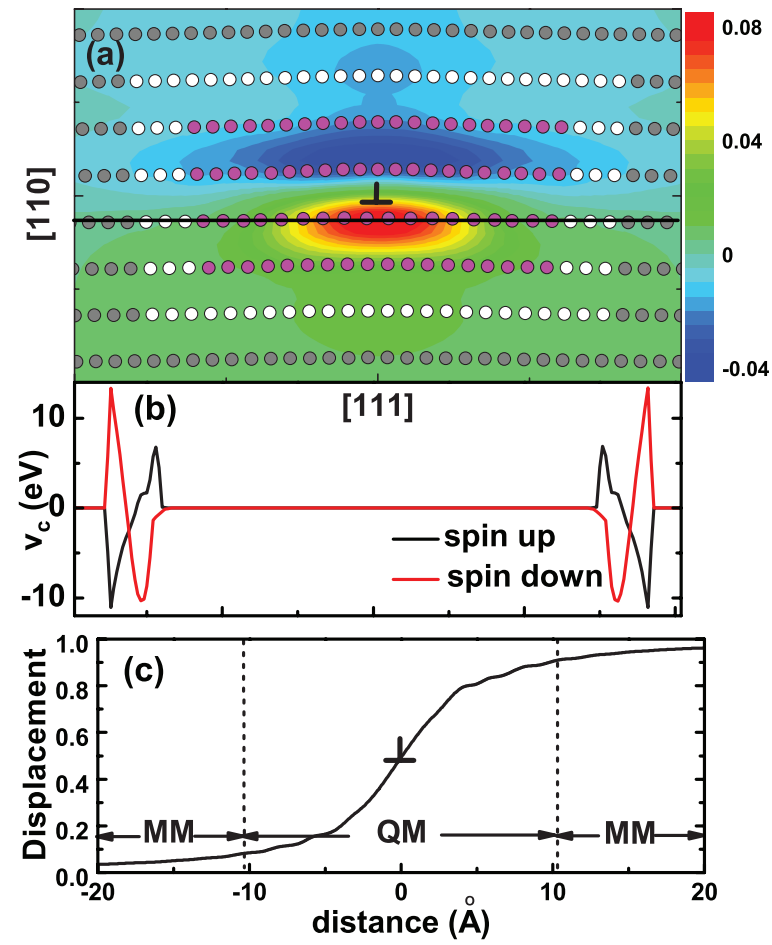

FIG. 6. (Color online) (a) The atomic structure and local strain field of the edge dislocation. The magenta, white, and gray circles represent the ions belonging to Regions I, II, and III, respectively. (b) The constraint potential along the horizontal line as indicated in (a). (c) The edge component of the displacement field in the unit of the Burgers vector for an edge dislocation in Fe determined from the $\mathrm{QM} / \mathrm{MM}$ method. The dislocation core is centered in the QM region and the dashed lines denote the QM/MM boundaries; only portions of the MM region are shown.

for the dislocation along a horizontal line. The general feature of the constraint potential is similar to that of the perfect $\mathrm{Al}$ lattice. As shown in Fig. 6(c), the QM/MM method can capture the correct long-range edge displacement of the dislocation, with a minimum of 0 and a maximum of $1 \mathbf{b}$ at the edges of the MM region. In a periodic DFT calculation, the simulation cell must contain either a dislocation dipole or quadrupole. ${ }^{44}$ The spreading of the Burgers vector shown in Fig. 6(c) demonstrates that a periodic DFT cell must be very large (about $80 \AA$ ) so that there is no overlap of the cores of the dipole or quadrupole dislocations. Use of a multiscale method captures the long-range tails of the Burgers vector distribution in the $\mathrm{MM}$ region while retaining full quantum resolution in the core region.

We note that the prediction of a dislocation core structure itself is only the first-level problem for computational materials science; it is really the computations of (i) chemical effects in the core due to solute alloying additions or embrittling species, (ii) structural changes in cores under applied loads or at finite temperatures, (iii) dislocation-dislocation interactions, and (iv) other dislocation/defect interactions such as dislocations precipitate interfaces that are of interest. Periodic DFT is far too expensive to use for these problems and the scope of possible cases (e.g., solute types, locations of embrittling agents, types of boundaries, etc.). Finally, the dislocation is just one lattice defect with long-range fields; another 


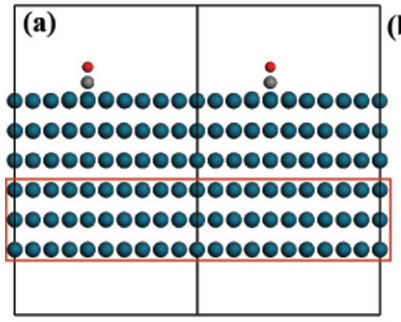

(c) :

000000000000000000000 000000000000000000000 000000000000000000000 $-000000000000000000000$ 000000000000000000000 000000000000000000000 -00000000000000000000 000000000000000000000

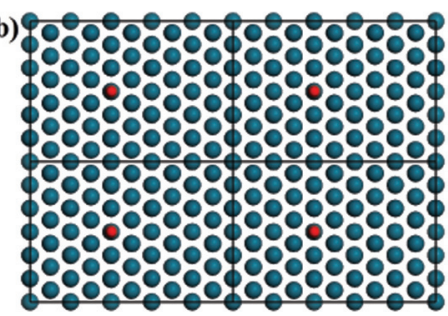

(d)

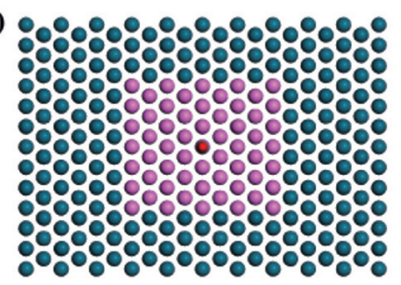

FIG. 7. (Color online) (a) The side view and (b) top view of the atomic structure in the stand-alone periodic DFT calculation. The black boxes represent the supercells and atoms within the red box are fixed during the atomic relaxation. (c) and (d) display the side and top view of the atomic structure in the QM/MM simulation. The $\mathrm{Pd}$ atoms are shown in blue in the stand-alone DFT calculations and are shown in magenta (blue) in the QM (MM) region. The gray and red spheres represent $\mathrm{C}$ and $\mathrm{O}$ atoms, respectively. The planes are $[1 \overline{1} 0] \times[111]$ and $[1 \overline{1} 0] \times[11 \overline{2}]$ for the side and top view, respectively.

case of high interest is a crack, for which there are no prospects whatsoever for periodic DFT calculations of any type.

\section{Chemisorption of $\mathrm{CO}$ on a flat/stepped $\operatorname{Pd}(111)$ surface}

$\mathrm{CO}$ oxidation is an important research area in catalysis because of its many important technological applications including exhaust gas after-treatment. ${ }^{46}$ Here, we calculate the chemisorption energies of a $\mathrm{CO}$ molecule on $\mathrm{Pd}$ flat and stepped surfaces to both validate and demonstrate the usefulness of the QM/MM method.

We first compare the chemisorption energy of $\mathrm{CO}$ on the flat $\operatorname{Pd}(111)$ surface between a stand-alone DFT and the QM/MM calculation. In the stand-alone DFT calculations, the Pd(111) surface is modeled by a six-layer slab of Pd atoms as shown in Fig. 7(a); the bottom three layers are fixed to their bulk positions while the top three layers are allowed to relax. In the $[1 \overline{1} 0] \times[11 \overline{2}]$ plane, the periodic supercell consists of $5 \times 2$ unit cells (120 Pd atoms). In all calculations, the energy cutoff of plane-wave basis is $400 \mathrm{eV}$ and the $k$-point mesh is $4 \times$

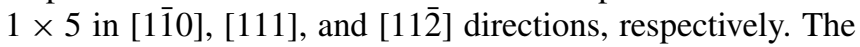
chemisorption energy is defined as

$$
\Delta E_{\mathrm{ad}}^{(a)}=E_{\mathrm{CO} / \mathrm{Pd}}^{(a)}-E_{\mathrm{Pd}}^{(a)}-E_{\mathrm{CO}}^{(a)},
$$

where $E_{\mathrm{CO} / \mathrm{Pd}}^{(a)}, E_{\mathrm{Pd}}^{(a)}$, and $E_{\mathrm{CO}}^{(a)}$ are the total energies of the chemisorbed surface, the clean Pd (111) slab, and the CO molecule, respectively. $\mathrm{CO}$ is found to preferentially adsorb on hollow sites of the Pd (111) surface, ${ }^{47,48}$ and the chemisorption energy $\Delta E_{\mathrm{ad}}^{(a)}$ is $-2.07 \mathrm{eV}$. For the QM/MM calculations, the entire system has $23100 \mathrm{Pd}$ atoms with the dimensions of $153.8 \AA, 47.9 \AA$ and $48.4 \AA$ in [110], [111], and [112]], respectively. The $\mathrm{QM}$ region consisting of $100 \mathrm{Pd}$ atoms is

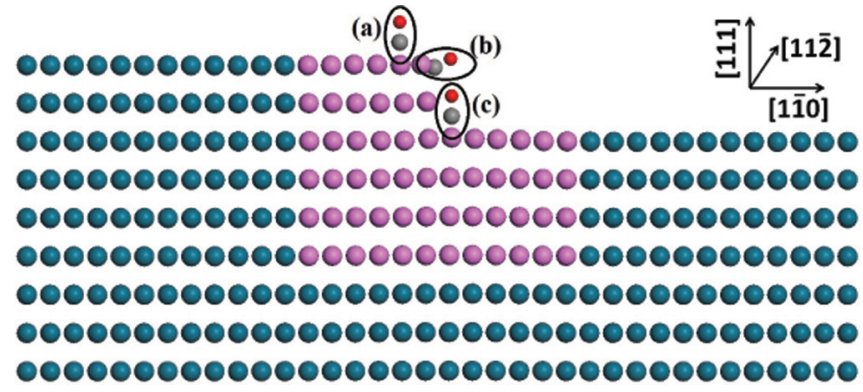

FIG. 8. (Color online) Atomic structure for modeling the chemisorption of a CO molecule on the Pd surface step. Three positions for the molecule on the step are indicated by the circles: (a) on the top surface, (b) on the step surface, and (c) on the lower surface.

placed at the center of (111) plane shown in Figs. 7(c) and 7(d). The chemisorption energy is calculated as

$$
\Delta E_{\mathrm{ad}}^{(b)}=E_{\mathrm{CO} / \mathrm{Pd}}^{(b)}-E_{\mathrm{Pd}}^{(b)}-E_{\mathrm{CO}}^{(a)}
$$

where $E_{\mathrm{CO} / \mathrm{Pd}}^{(b)}$ and $E_{\mathrm{Pd}}^{(b)}$ are the total energies of the entire system with and without the absorbed $\mathrm{CO}$ molecule, respectively. The chemisorption energy $\Delta E_{\mathrm{ad}}^{(b)}$ is $-2.09 \mathrm{eV}$, comparing very well to the stand-alone DFT value. This comparison again validates the energetics of the QM/MM method.

Because of the importance of surface steps in catalysis, we examine the chemisorption energy of $\mathrm{CO}$ at three adsorption sites near a $\mathrm{Pd}$ step as shown in Fig. 8. The QM/MM method is ideal for treating surface steps because it can mitigate the fictitious step-step interactions in the periodic DFT calculations.

The surface step is modeled by removing two atomic layers on the (111) plane and a periodic boundary condition is applied in the [112] direction. The QM/MM system measures $166.4 \AA \times 134.7 \AA \times 9.69 \AA$ with $14160 \mathrm{Pd}$ atoms in total. The dimensions of the $\mathrm{QM}$ region are $15.35 \AA \times 14.10 \AA \times 9.69 \AA$ with $120 \mathrm{Pd}$ atoms. A $1 \times 1 \times 5 k$-mesh is used. Following Eq. (20), the chemisorption energies are: $-2.05 \mathrm{eV},-2.04 \mathrm{eV}$, and $-1.63 \mathrm{eV}$ for the adsorption sites (a), (b), and (c), respectively. While the sites (a) and (b) have the similar chemisorption energy as the flat surface, the site (c) lowers the chemisorption energy by $0.4 \mathrm{eV}$ as compared to the flat surface. This example shows that the chemisorption energy depends sensitively on the local structure of the adsorption sites, particularly near surface defects such as steps and edges. This is where the QM/MM method could be particularly useful.

\section{CONCLUSION}

We have introduced a $\mathrm{QM} / \mathrm{MM}$ method that is based on the concept of constrained DFT to capture the nonadditive kinetic energy that is usually missing in current QM/MM methods using Kohn-Sham DFT. The implementation of the QM/MM method, including the construction of the bulklike charge density, the calculation of the constraint potential and the optimization of the penalty parameter, have been discussed. Sources of error in the method are identified, all of which can be reduced without fundamental changes to the method itself. The QM/MM method has been applied to three different types 
of metals (Al, Fe, and Pd) and is validated against standard periodic DFT calculations. For the perfect bulk lattice of Fe, we show that the QM/MM method can reproduce the magnetic moments of the periodic DFT calculations with good accuracy. The QM/MM method also predicts the vacancy formation energy of $\mathrm{Al}$, the magnetic moments at a self-interstitial atom of $\mathrm{Fe}$, and the chemisorption of $\mathrm{CO}$ on the (111) surface of $\mathrm{Pd}$ quite well, in comparison to periodic DFT results. We have also applied the QM/MM method to predict the core structure of the edge dislocation in $\mathrm{Fe}$, where periodic DFT calculations are not possible without significant errors due to the necessity of using small cell sizes. Finally, we used the QM/MM method to examine the chemisorption energy of $\mathrm{CO}$ on a stepped Pd surface, pointing toward future applications of the method in catalysis. In general, this QM/MM method has the same level of accuracy and efficiency as the OFDFT-based QM/MM embedding method, which is limited to main group elements. The present method has no inherent restrictions on application across the periodic table, within the limits of application of Kohn-Sham DFT methods, and can deal with arbitrary geometries including nonperiodic systems. As such, it is a valuable tool for performing accurate first-principles calculations for problems that might otherwise not be computationally feasible.

\section{ACKNOWLEDGMENT}

We acknowledge support of this work by the Army Research Office through the MURI program "Stress-Controlled Catalysis via Engineered Nanostructures," W911NF-11-10353. *ganglu@ csun.edu

${ }^{1} \mathrm{G}$. Lu and E. Kaxiras, in Handbook of Theoretical and Computational Nanotechnology, edited by M. Rieth and W. Schommers (American Scientific, Stevenson Ranch, CA, 2004), Chap. 22.

${ }^{2}$ N. Bernstein, J. R. Kermode, and G. Csanyi, Rep. Prog. Phys. 72, 026501 (2009).

${ }^{3}$ H. Lin and D. G. Truhlar, Theor. Chem. Acc. 117, 185 (2007).

${ }^{4}$ I. Antes and W. Thiel, ACS Symp. Ser. 712, 50 (1998).

${ }_{5}^{5}$ J. Gao and D. G. Truhlar, Annu. Rev. Phys. Chem. 53, 467 (2002).

${ }^{6}$ P. Huang and E. A. Carter, Annu. Rev. Phys. Chem. 59, 261 (2008).

${ }^{7}$ X. Zhang, Y. Zhao, and G. Lu, Int. J. Multiscale Comput. Eng. 10, 65 (2012).

${ }^{8}$ A. K. Nair, D. H. Warner, R. G. Hennig, and W. A. Curtin, Scr. Mater. 63, 1212 (2010).

${ }^{9}$ C. Woodward and S. I. Rao, Phys. Rev. Lett. 88, 216402 (2002).

${ }^{10} \mathrm{P}$. Suryanarayana, V. Gavini, T. Blesgen, K. Bhattacharya, and M. Ortiz, J. Mech. Phys. Solids 58, 256 (2010).

${ }^{11}$ S. Ismail-Beigi and T. A. Arias, Phys. Rev. Lett. 82, 2127 (1999).

${ }^{12}$ N. Choly, G. Lu, W. E, and E. Kaxiras, Phys. Rev. B 71, 094101 (2005).

${ }^{13}$ X. Zhang and G. Lu, Phys. Rev. B 76, 245111 (2007).

${ }^{14}$ X. Zhang, C. Y. Wang, and G. Lu, Phys. Rev. B 78, 235119 (2008).

${ }^{15}$ P. Cortona, Phys. Rev. B 44, 8454 (1991).

${ }^{16}$ T. A. Wesolowski and A. Warshel, J. Phys. Chem. 97, 8050 (1993).

${ }^{17}$ N. Govind, Y. A. Wang, A. J. R. da Silva, and E. A. Carter, Chem. Phys. Lett. 295, 129 (1998).

${ }^{18}$ P. Huang and E. A. Carter, J. Chem. Phys. 125, 084102 (2006).

${ }^{19}$ M. Hodak, W. Lu, and J. Bernholc, J. Chem. Phys. 128, 014101 (2008).

${ }^{20}$ X. Zhang, Q. Peng, and G. Lu, Phys. Rev. B 82, 134120 (2010).

${ }^{21} \mathrm{Q}$. Wu and T. Van Voorhis, Phys. Rev. A 72, 024502 (2005).

${ }^{22}$ O. Roncero, M. P. de Lara-Castells, P. Villarreal, F. Flores, J. Ortega, M. Paniagua, and A. Aguado, J. Chem. Phys. 129, 184104 (2008).

${ }^{23}$ J. D. Goodpaster, N. Ananth, F. R. Manby, and T. F. Miller III, J. Chem. Phys. 133, 084103 (2010).

${ }^{24}$ S. Fux, C. R. Jacob, J. Neugebauer, L. Visscher, and M. Reiher, J. Chem. Phys. 132, 164101 (2010).
${ }^{25}$ C. Huang, M. Pavone, and E. A. Carter, J. Chem. Phys. 134, 154110 (2011).

${ }^{26}$ Q. Zhao and R. G. Parr, J. Chem. Phys. 98, 543 (1992).

${ }^{27}$ Q. Zhao, R. C. Morrison, and R. G. Parr, Phys. Rev. A 50, 2138 (1994).

${ }^{28}$ Q. Wu and W. Yang, J. Chem. Phys. 118, 2498 (2003).

${ }^{29}$ E. B. Tadmor, M. Ortiz, and R. Phillips, Philos. Mag. A 73, 1529 (1996).

${ }^{30}$ M. S. Daw and M. I. Baskes, Phys. Rev. B 29, 6443 (1984).

${ }^{31}$ Q. Peng, X. Zhang, L. Hung, E. A. Carter, and G. Lu, Phys. Rev. B 78, 054118 (2008).

${ }^{32}$ W. Kohn, Int. J. Quantum Chem. 56, 229 (1995).

${ }^{33}$ P. Hohenberg and W. Kohn, Phys. Rev. 136, B864 (1964).

${ }^{34}$ G. Kresse and J. Hafner, Phys. Rev. B 47, 558 (1993).

${ }^{35}$ G. Kresse and J. Furthmuller, Phys. Rev. B 54, 11169 (1996).

${ }^{36}$ P. E. Blochl, Phys. Rev. B 50, 17953 (1994).

${ }^{37}$ J. P. Perdew and A. Zunger, Phys. Rev. B 23, 5048 (1981).

${ }^{38}$ J. P. Perdew, K. Burke, and M. Ernzerhof, Phys. Rev. Lett. 77, 3865 (1996).

${ }^{39}$ H. J. Monkhorst and J. D. Pack, Phys. Rev. B 13, 5188 (1976).

${ }^{40}$ F. Ercolessi and J. Adams, Europhys. Lett. 26, 583 (1994).

${ }^{41}$ V. Shastry and D. Farkas, Simul. Mater. Sci. Eng. 4, 473 (1996).

${ }^{42}$ H. W. Sheng, M. J. Kramer, A. Cadien, T. Fujita, and M. W. Chen, Phys. Rev. B 83, 134118 (2011).

${ }^{43}$ C. Huang and E. A. Carter, Phys. Chem. Chem. Phys. 10, 7109 (2008).

${ }^{44}$ J. R. K. Bigger, D. A. McInnes, A. P. Sutton, M. C. Payne, I. Stich, R. D. King-Smith, D. M. Bird, and L. J. Clarke, Phys. Rev. Lett. 69, 2224 (1992).

${ }^{45}$ L. Ventelon and F. Willaime, J. Comput. Aided Mater. Des. 14, 85 (2007).

${ }^{46}$ T. Engel and G. Ertl, in The Chemical Physics of Solid Surfaces and Heterogeneous, edited by D. A. King and D. P. Woodruff, Vol. 4 (Elsevier, New York, 1982).

${ }^{47}$ B. Hammer, L. B. Hansen, and J. K. Norskov, Phys. Rev. B 59, 7413 (1999).

${ }^{48}$ C. J. Zhang and P. Hu, J. Am. Chem. Soc. 123, 1166 (2001). 